\title{
Lidil
}

Revue de linguistique et de didactique des langues

$36 \mid 2007$

Échanges exolingues via internet et appropriation des langues-cultures

\section{La variation sociale en français}

Gadet Françoise, 2007, 2édition, Paris, Ophrys.

\section{Cyril Trimaille}

\section{OpenEdition}

\section{Journals}

Édition électronique

URL : http://journals.openedition.org/lidil/2583

DOI : $10.4000 /$ lidil. 2583

ISSN : $1960-6052$

Éditeur

UGA Éditions/Université Grenoble Alpes

Édition imprimée

Date de publication : 1 décembre 2007

ISBN : 978-2-84310-108-3

ISSN : $1146-6480$

Référence électronique

Cyril Trimaille, « La variation sociale en français », Lidil [En ligne], 36 | 2007, mis en ligne le 01 juin 2009, consulté le 22 septembre 2020. URL : http://journals.openedition.org/lidil/2583 ; DOI : https:// doi.org/10.4000/lidil.2583

Ce document a été généré automatiquement le 22 septembre 2020.

(C) Lidil 


\title{
La variation sociale en français
}

\author{
Gadet Françoise, 2007, 2édition, Paris, Ophrys.
}

\section{Cyril Trimaille}

1 Comme la précédente, cette deuxième édition revue et augmentée se propose de décrire le français contemporain, de s'interroger sur sa spécificité et de mettre ces éléments en perspective avec les débats et enjeux qui traversent non seulement la sociolinguistique mais aussi la linguistique générale actuelles. Ce faisant, F. Gadet réaffirme la pertinence du rapprochement entre linguistique et sociolinguistique. Le parti pris de l'auteure est, comme dans nombre de ses écrits, de décrire différentes dimensions des variations $d u$ français, en partant des pratiques langagières authentiques de sujets (et non seulement de locuteurs), saisies en contexte.

2 Pour développer cette perspective "écologique " sur le français et ses usages, la structure du livre reste identique à celle de la précédente édition. Six chapitres, tous prolongés de bibliographies thématiques, de pistes d'approfondissements variées (pour aller plus loin) et d'orientations bibliographiques. La plupart des sections descriptives et analytiques sont ponctuées d'études de cas et enrichies de développements intitulés outils d'analyses, consacrés à des aspects théoriques ou méthodologiques. Les six chapitres sont en outre complétés par des suggestions d'exercices, un glossaire d'une centaine de notions, une bibliographie générale et un index, qui sont autant d'outils d'approfondissement fort utiles.

3 Le premier chapitre est une entrée en matière qui pose, pour toute langue, le caractère fondamentalement constitutif de l'hétérogénéité. F. Gadet y définit, exemples à l'appui, les principes extra-linguistiques qui concourent à configurer ou structurer cette diversité : les dimensions diachroniques, géographiques, sociales et situationnelles (parmi lesquelles le diamésique). L'auteure y évoque le rôle précurseur de la dialectologie dans l'étude de la diversité linguistique et les nouvelles potentialités offertes par le développement des grands corpus, tout en appelant à une grande rigueur dans toutes les procédures, de leur constitution à leur exploitation. Elle part ensuite de la réception (perception et évaluation) des phénomènes variationnels, souvent en termes de variétés qui, bien que rarement nommées, ont tendance à essentialiser des usages toujours labiles, pour aboutir à une discussion des 
"catégorisations d'experts". Plaidant, à la suite de Coseriu, pour une approche variationnelle de la langue, elle pointe les carences à la fois des linguistiques de l'homogène et de l'appréhension de l'hétérogénéité en variétés, qui déplacent à un autre niveau la recherche de l'homogénéité perdue.

F. Gadet définit ensuite deux types de normes, objective (statistique) et subjective (La Norme, imposée). Si l'on ne peut qu'être d'accord avec la nécessité de distinguer entre ces deux types de normes, on peut néanmoins discuter le choix de l'adjectif subjectif pour référer à ce que d'autres (notamment Rey 19721) nomment norme prescriptive, réservant le qualificatif subjectif à la conscience normative des locuteurs. En lien avec ces questions de norme et de standardisation, F. Gadet conclut le chapitre en appréhendant le poids de l'idéologie du standard qui a conduit à ce purisme si typiquement français.

5 Le deuxième chapitre est consacré aux relations entre oral et écrit. L'auteure s'y intéresse à l'observation et à la transcription de l'oral dont les structures, adaptées aux contraintes mais aussi aux potentialités matérielles et situationnelles (co-présence, multimodalité liée à la "vive voix ", co-énonciation), apparaissent encore souvent, à tort, lacunaires ou défectueuses. Cela soulève nombre de questions cruciales à propos de la représentation de l'oral par les linguistes. F. Gadet montre encore que ces questions, d'apparence méthodologique, sont en fait inséparables de la conception théorique que l'on a des rapports oral/écrit. Appelant notamment à remettre en cause l'hypothèse d'un "grand partage", elle fait état de différentes tentatives de dépassement les clivages binaires de type simple vs complexe (renvoyant insidieusement au clivage nature/culture) en présentant les approches de Halliday, de Koch \& Esterreicher, ou encore celle de Biber qui privilégie la notion de registres (proche des genres bakhtiniens) à celle d'une dichotomie médiale, c'est-à-dire liée uniquement au medium.

6 Le chapitre suivant traite $d u$ "matériau variationnel ", autrement dit des zones $d u$ français, selon F. Gadet en nombre limité, touchées par la variation que l'on peine souvent à appréhender hors de la prégnance du standard et de l'écrit (cf. les termes de réduction, simplification, relâchement).

7 L'examen du plan phonique (segmental et supra-segmental) permet d'introduire la notion de saillance et de passer en revue quelques-unes des variables les plus investies sociolinguistiquement en français hexagonal (schémas prosodiques, pauses, 'e' muet, simplification de groupes consonantiques, liaisons, etc.). L'observation de ces variables débouche sur une typologie de la variation phonique. Bien que moins saillantes, les variantes morphosyntaxiques constituent un réservoir de stéréotypes sociolinguistiques (dont un prototype pourrait être aller au docteur). Mais plutôt que de conserver l'opposition standard vs non-standard, l'auteure propose d'appréhender la variation morpho-syntaxique en termes de registres de distance ou de proximité, respectivement caractérisés par une plus ou moins grande décontextualisation.

8 Les dynamiques variationnelles des deux plans, phoniques et grammaticaux, sont ensuite examinées à la lumière des arguments fonctionnalistes de Kroch (pour qui la volonté de distinction sociale des élites les amènerait à inhiber des tendances évolutives), de Frei (selon qui les 'fautes' répondraient à des besoins linguistiques) ou encore de Berrendonner (qui insiste sur les fonctions pragmatiques de désambigüisation, de résolution de conflits de sens). Enfin, sont présentés et discutés 
les liens entre variation et changement linguistique, ainsi que quelques changements en cours (e prépausal, liaison sans enchainement).

9 L'auteure ouvre le quatrième chapitre en présentant différents concepts référant à des agrégats ou unités sociales utilisés en sociolinguistique. Elle distingue ceux utilisés par les linguistes - contexte, communauté - de ceux issus de la sociologie - marché linguistique, réseau social, interaction - et qui privilégient des aspects plus relationnels ainsi qu'un lien micro-macro. On peut s'interroger sur le fait que n'apparaisse pas, dans cette présentation de concepts issus de la sociologie, la notion de communauté de pratiques, cette absence pouvant sans doute s'expliquer par sa non utilisation en sociolinguistique francophone.

10 La deuxième partie du chapitre traite de l'étude du poids de facteurs externes dans le changement linguistique, des questions soulevées par le paradigme variationniste, et discute du prestige (manifeste ou latent) comme moteur de diffusion des innovations.

11 F. Gadet expose ensuite plusieurs études de cas qui touchent la socialisation langagière, la transmission scolaire des normes et les éventuels ressorts sociolinguistiques de l'échec scolaire, revenant notamment sur les travaux de Lahire, Dannequin ou Labov, et ceux, controversés, de Bernstein.

12 L'étude des vernaculaires fait l'objet du chapitre 5, dans lequel F. Gadet aborde différents aspects du français populaire, s'interroge sur la catégorie langue des jeunes (deux de ses domaines privilégiés), et thématise le contact des langues induit par l'immigration. La problématique commune à ces trois objets, incontestablement connexes, semble toucher les questions des frontières entre "variétés " et des désignations problématiques de ces dernières.

13 Un autre apport majeur de l'ouvrage se situe sans doute dans le sixième et dernier chapitre dans lequel F. Gadet fait un état de l'art critique de la question et des théories $\mathrm{du}$ diaphasique, synthèse qui faisait défaut en langue française. Elle y offre une nouvelle formulation de son hypothèse selon laquelle, sous l'influence d'évolutions sociétales, la variation du français de France serait successivement passée d'une dominance du diatopique, à une prépondérance diastratique pour arriver aujourd'hui au primat du diaphasique.

14 Soulignant, une fois encore, l'inadéquation empirique des catégories telles que niveaux ou registres de langues, l'auteure rappelle qu'en dépit des représentations communes, tout locuteur natif, même défavorisé, est doué d'une certaine souplesse diaphasique. Cette variation intra-locuteur peut aller jusqu'à donner la forme d'« incongruences » stylistiques à certaines occurrences "dont l'apparent désordre constitue une objection à l'idée même de variété qui se montre alors pour ce qu'elle est, une abstraction » (p. 152).

15 Au total, la diversité des thèmes traités, la documentation minutieuse (empirique et scientifique), la clarté et l'organisation spiralaire permettant l'abord de notions et de problématiques par touches successives et sous différents angles, font de ce livre un auxiliaire précieux tant pour des étudiants devant entrer dans la problématique de la variation que pour des chercheurs souhaitant en approfondir certains aspects théoriques ou empiriques. Si, force est de le reconnaitre, la sociolinguistique n'est pas (toute) la linguistique, F.Gadet montre une fois encore combien ce mode d'appréhension des réalités langagières dans leur complexité, interne et externe, peut apporter à leur compréhension. 


\section{NOTES}

1. Alain Rey, 1972, « Usages, jugements et prescriptions linguistiques, Langue française 16, 4-28.

\section{AUTEUR}

\section{CYRIL TRIMAILLE}

Université Stendhal-Grenoble 3, Lidilem. 